\title{
Three-Dimensional Nanoscale Manipulation and Manufacturing using Proximal Probes: Controlled Pulling of Polymer Micro/Nanofibers
}

\author{
Amrinder S. Nain ${ }^{\dagger \ddagger}$, Cristina Amon ${ }^{\dagger \ddagger}$ and Metin Sitti ${ }^{\dagger+\ddagger}$ \\ Department of Mechanical Engineering ${ }^{\dagger}$, Robotics Institute ${ }^{*}$ and Institute for Complex Engineered Systems ${ }^{\ddagger}$ \\ Carnegie Mellon University, Pittsburgh, PA 15213-3890 \\ \{anain,camon,msitti\}@andrew.cmu.edu
}

\begin{abstract}
Besides imaging and characterization, proximal probes are proposed to be used as three-dimensional (3D) nanoscale manipulation and manufacturing tools in this paper. We propose 3D nanoscale pulling of liquid polymer micro/nanofibers by precise positioning of Atomic Force Microscope (AFM) nanoprobes and control of polymer solidification. An AFM probe is used to pull or extrude thermoset and thermoplastic polymers precisely to fabricate 3D polymer nano-fiber structures. A liquid polymer fiber bridge between the probe tip and a substrate is maintained when pulling the probe from the surface with controlled speed and position. We present results of our pulling experiments in vertical, horizontal and arbitrary 3D pulling directions for Poly(methyl methacrylate): PMMA polymer fibers. Force-distance curves obtained using AFM for PMMA samples at different scan rates are presented. A preliminary study showing the effect of velocity profile on pulling liquid bridges using POLYFLOW ${ }^{(3)}$ is presented.
\end{abstract}

Keywords-Nanomanipulation, Nanomanufacturing, Nanorobotics

\section{INTRODUCTION}

One of the most significant barriers for enabling the breakthroughs promised by nanotechnology, is mass production of nanoscale structures, devices, and systems. Therefore, novel manufacturing processes at the micro/nanoscale are indispensable for the commercialization of future nanoscale devices, circuits, man-made materials, sensors, etc. One of the main challenges of nanomanufacturing systems is $3 \mathrm{D}$ customized manufacturing. This work aims to fill this gap by scaling the traditional fiber pulling-based manufacturing technology to the micro/nanoscale for the first time. Atomic Force Microscope (AFM) proximal probes are used to manufacture customized three-dimensional (3D) polymer nano-fibers by controlled pulling and solidification of liquid polymers. For this goal, the fundamental issues of surface chemistry of the probe, liquid polymer curing, polymer fiber aspect ratio (length/diameter), fiber orientation in 3D, rheology and heat transfer at the micro/nanoscale are critical. This micro/nano-fiber pulling technology would have wide applications in nano-circuit interconnects by using conductive nano-fibers and prototyping novel nano-electronic devices by using conductive/semiconductor/non-conductive polymer fiber structures, 3D polymer fiber-based nano-actuators, photonic devices, novel bio-nano-sensors, smart materials, etc.

The use of polymers is not new, and they have been studied in detail [1-3]. However, there is a thrust to develop and study polymers at the nanoscale [4-7]. This pulling-based micro/nano-fiber manufacturing technique has many merits as compared to other current nano-manufacturing techniques:

- It enables precise and three-dimensional customized polymer fibers down to the nanometer scale, where there is no such method yet in the literature.

- It is possible to manufacture conducting, non-conducting, and semi-conducting polymer fibers in the same process.

- Multi-length scale fibers can be pulled by controlling the pulling probe shape; thus, it is possible to integrate multilength scale fiber manufacturing in the same system.

- Using the real-time force detection of the AFM probe, closed-loop control automatic pulling schemes can be developed.

- Massively parallel fiber manufacturing is possible by an array, e.g. thousands, of AFM probes in parallel [8] with independent fiber pulling control.

- The overall system can be miniaturized by using selfsensing, e.g. piezoresistive sensing type, AFM probes, sensors, on-board control and driving circuitry, power sources, and actuators. This would lead to compact and cost-effective nano-manufacturing systems.

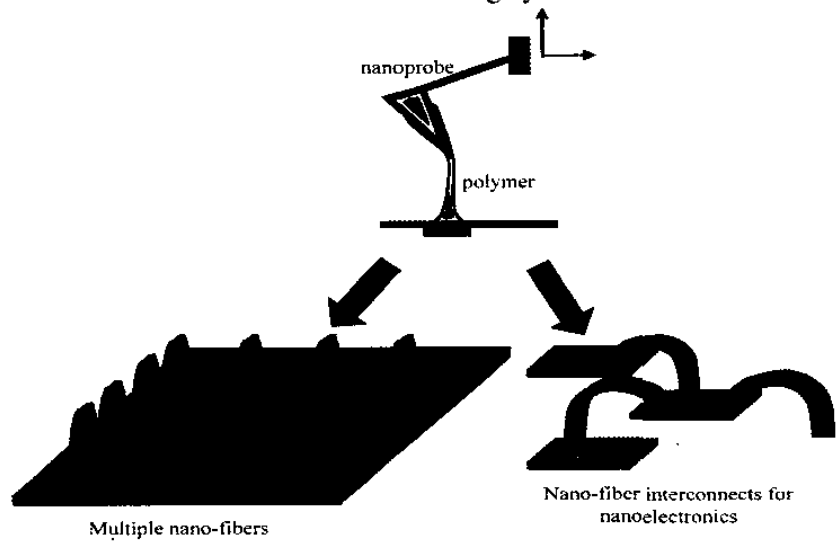

Figure1. The concept of Atomic Force Microscope probe based polymer pulli and solidification in real-time

The general scheme of pulling liquid polymers using an AFM probe for pulling nano-pillars and electronic circuit interconnects is depicted in Figure 1.

\section{PROBLEM DEFINITION}

The initial goals of the project entail validating the proof of the concept of pulling the polymer fibers in multiple modes: vertical, horizontal, and in random three-dimensional trajectories. This is to be followed by developing a control 
loop for pre-determined trajectories. The proposed technique has not been tested and the feasibility has not yet been shown for controlled micro/nanoscale polymer trajectories. At reduced lengthscales, the very high surface-to-volume ratio of the polymer micro/nano-fiber requires determining several

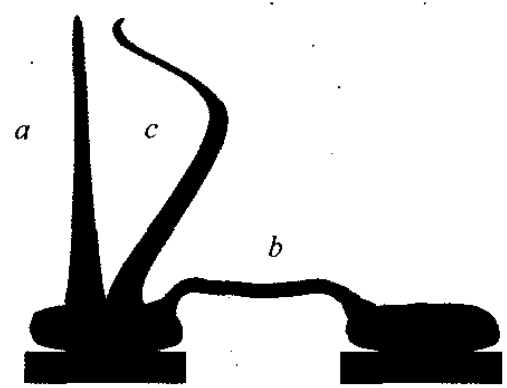

Figure 2. (a) Vertical fiber, (b) Horizontal fiber bridging two polymer

unknown parameters, such as the surface chemistry of the probe, wetting behavior, rheology, heat transfer, kinetics of crystallization, and perhaps molecular chain orientations. Nanolithography techniques such as Dip Pen Lithography [9] are geared towards depositing material on the substrate and/or patterning the substrate in $2 \mathrm{D}$, whereas our approach seeks to develop high aspect ratio polymer fibers anchored to the substrate. Polymer fibers can be pulled in three dimensions (Figure 2) where the AFM tip approaches a substrate (with the

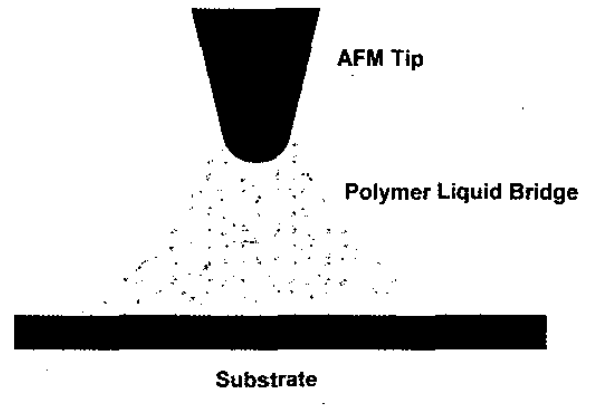

Figure 3. Polymer Liquid-Bridge formed initially

polymer solution in the form of a droplet on the substrate) and subsequently retracts, thus forming a fiber through an initial liquid bridge (Figure 3).

Polymer Classification and Pulling Strategies

The choice of polymer dictates the way the polymer is pulled using AFM. We have classified the polymers under three main categories:

1. Liquid Polymer Mixtures: Some viscous liquid polymers are mixed with a solvent at room temperature and deposited on the substrate. The AFM tip is brought into contact with the substrate and the fiber is pulled. With time, due to the high surface-to-volume ratio at the micro/nanoscale, the solvent evaporates, thus forming a continuous fiber. Thus, no heating or cooling is required for these types of polymers for solidification.
2. Thermoplastic Polymers: These polymers are solid at room temperature and can be melted and solidified reversibly by heating and cooling. They are spun on a substrate or deposited on a nanoprobe. Pulling is achieved either by heating the probe or heating the substrate using a resistive heater element, so as to form a liquid polymer that can be pulled. Once the liquid bridge is formed, solidification of the polymer is achieved by heat transfer to the environment from the liquid bridge.

3. Thermoset Polymers: These polymers are liquid at room temperature, and solidified irreversibly by heating. They are spun on a substrate or deposited on a nanoprobe. After forming the liquid bridge, the substrate is heated or UV radiation is used to solidify the polymer fiber.

The authors previously presented [10] a strategy for pulling micro/nano-fibers from thermoset resins. In our earlier studies, the choice between using thermoplastics or thermosetting resins was out of convenience. Thermosetting polymers are available in liquid form at room temperature; thus, we could devise quick experiments to validate our initial thoughts. On the other hand, thermoplastics can be used easily by heating the probe tip, which can be accomplished by passing current through a doped silicon AFM probe or by attaching the substrate to a heater element.

\section{EXPERIMENTAL METHODS}

\section{Experimental Setup and Polymers Used}

For our experiments, we made use of a Park Scientific Instruments Autoprobe M5 with Park Scientific Instruments Contact (Stiffness $\geq 20 \mathrm{~N} / \mathrm{m}$ ) and Non-Contact Ultralevers mounted (Stiffness $\leq 1 \mathrm{~N} / \mathrm{m}$ ). We also used Scanning Tunneling Microscope (STM) probes for pulling liquid polymer mixtures. The polymer used was a solution of PMMA photo resist with a $950 \mathrm{~K}$ molecular weight dissolved in either anisole or chlorobenzene in different concentrations by weight. While our experimental setup (Figure 4) has the capability of integrating a haptic device, this study did not use the haptic interface. We envision designing a fresh set of experiments in line with previous work $[11,12]$ in the near
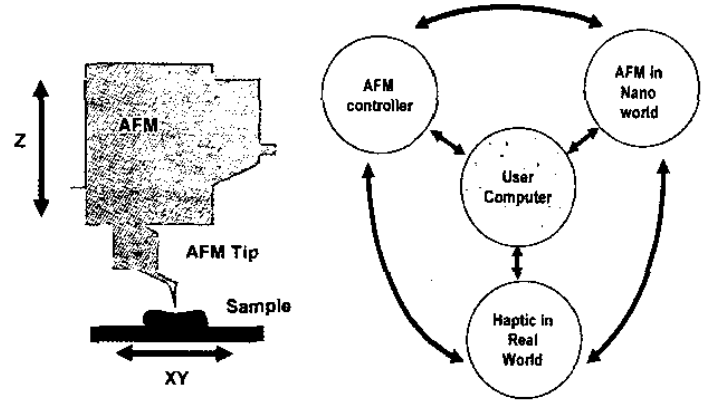

Figure 4. AFM Schematic and AFM control

future.

\section{Fiber Pulling Strategy}

In our experiments, we deposited a polymer droplet on a silicon substrate and the AFM probe tip was lowered until contact with the droplet was established. A liquid bridge was 
formed as shown in Figure 5, the height of which varied with the contact depth of the AFM tip and polymer solution. As the AFM tip was retracted, the liquid polymer bridge reduced in cross section and increased in length until failure. In a similar approach, polymer fibers have been pulled [13] which are significantly larger than the fibers this research aims to pull. No-slip conditions both at the substrate and the tip surface

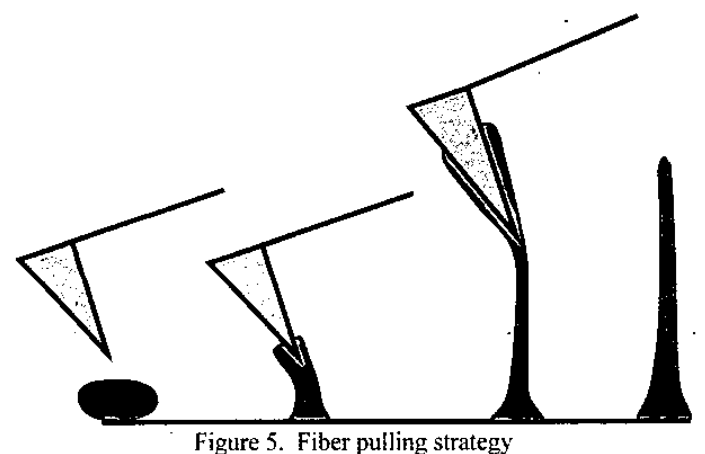

tend to form fibers that are larger at the base and narrow at the apex. This fiber pulling strategy, though straightforward, is complicated due the following problems:

- Wetting of the polymer on the AFM probe needs to be controlled, as it leads to formation of large undesirable fibers and sometimes loss of tips due to the viscoelasticity of the polymer solution.

- After the formation of the fiber, the polymer is deposited on the AFM probe tip for subsequent trials and this leads to subsequently larger fibers.

- Fibers pulled using the same strategy, but in the horizontal direction exhibit sagging due to their weight at the mid-plane.

- The solvent in the solute-solvent polymer solution evaporates thus creating a limited window of opportunity within which long fibers can be pulled. Before this opportunity, the solution is too fluidic to be pulled and after the opportunity, it is too dry.

\section{RESULTS}

\section{Vertical Direction}

Our initial experiments comprised PMMA-anisole solution droplets on a silicon substrate, from which Non-Contact AFM

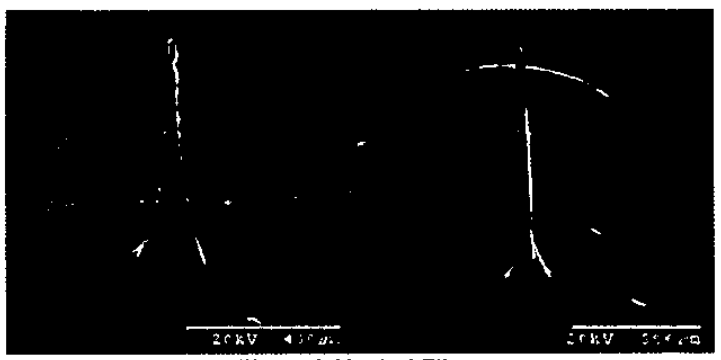

Figure 6. Vertical Fibers

Ultralevers were used to draw fibers in vertical orientations
(Figure 6). The length of the fibers depended upon the length of time the solvent had evaporated and the relative concentrations of PMMA and anisole. In a subsequent paper, we plan on presenting results on the aspect ratio of fiber achieved as a function of different polymer-solvent combinations and concentrations.

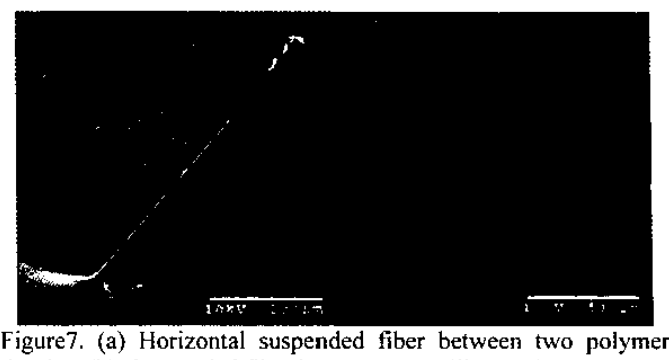

droplets (b). Suspended fiber between two silicon substrates

\section{Horizontal Direction}

Throughout our first such experiments, two parallel lines of polymer solution were deposited on the substrate and fibers were pulled between them. We observed that fibers pulled across the plane often tended to rest upon the substrate, even when they were drawn up several microns before being drawn across the substrate, indicating that the load of the fiber's weight was pulling the fibers down to the substrate.

Moving the polymer lines closer together allowed some wires to remain suspended above the substrate in their orientations (Figure 7), but a more novel solution involved two adjacent pieces of silicon with a gap between them of only a few hundred microns. With concurrent successes, it became a simple matter to draw the fibers into whatever orientation across the plane was desired (Figure 8(a)), showing a cross formation. Subsequently, we were able to pull multiple horizontal fibers across two substrates (Figure $8(\mathrm{~b})$ ).

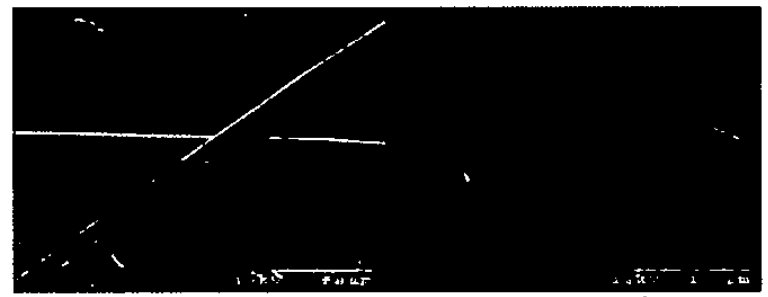

Figure8. (a) Criss-cross pattern (b) Multiple horizontal fibers

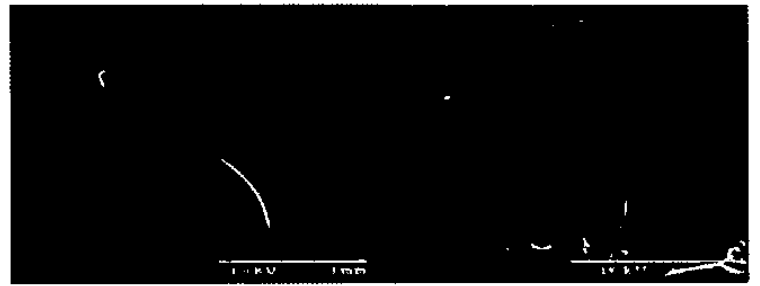

Figure 9. Three Dimensional Fibers 


\section{Three-Dimensional Trajectories}

Combining the methods of horizontal and vertical pulling yielded fibers which maintained a three-dimensional suspended geometry (Figure 9) according to the orientation into which the fiber was pulled. Future experiments will test and refine methods for controlling the 3-D pulling of fibers into specific shapes and orientations.

\section{Fibers of Special Interest}

In Figure (10 a-d ), we show the first sub-micron fiber fabricated in our group using an STM tip. Figure $11(a)$ is a

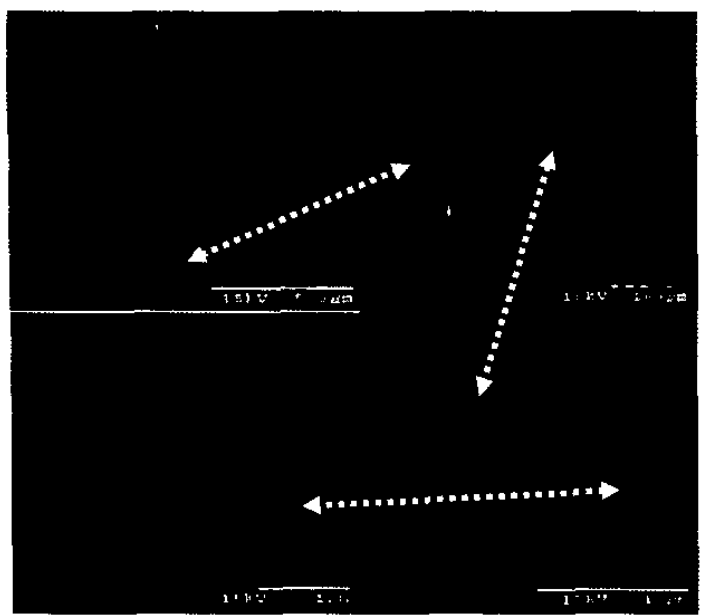

Figure 10. Sub-micron fiber

vertical smooth fiber we were able to pull and $11(\mathrm{~b})$ is a fiber showing possible instability, hence the peculiar structure. This instability could be due to intrinsic polymer behavior or the fiber pulling strategy. We are currently determining the cause of this structure.

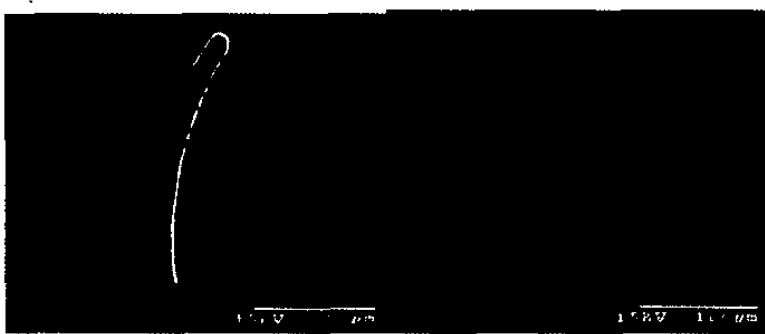

Figure 11. (a) Vertical Smooth Fiber, (b) Polymer fiber showing possible instability

\section{MODELING FIBER PULLING}

We envision that modeling the nano-fiber pulling system involves solidification reaction, viscoelastic mechanics, surface tension (wetting), heat transfer, and crystallization kinetics modeling as illustrated in Figure 12. Many of the constants at these length scales are unknown and we are currently in the process of investigating continuum and sub- continuum effects. An accurate determination of these constants is a pre-requisite towards modeling these length scales.

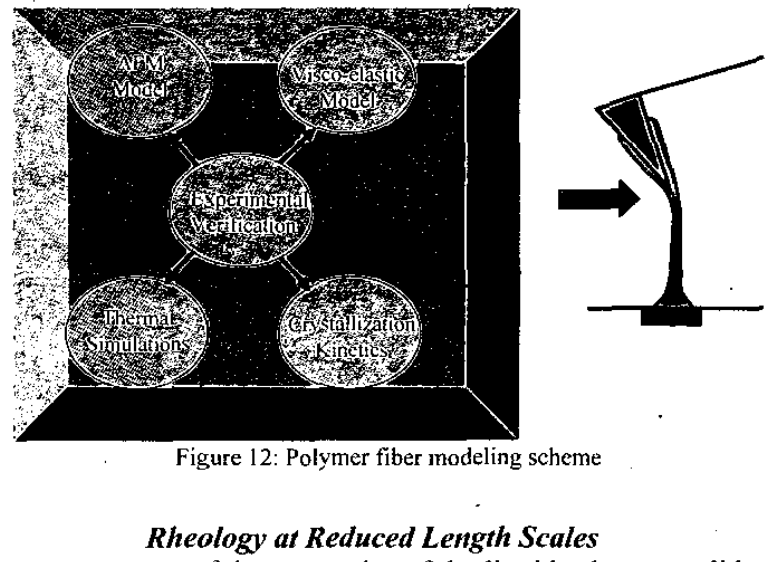

The measurement of the properties of the liquids close to solid surfaces-or confined in narrow spaces is important in various

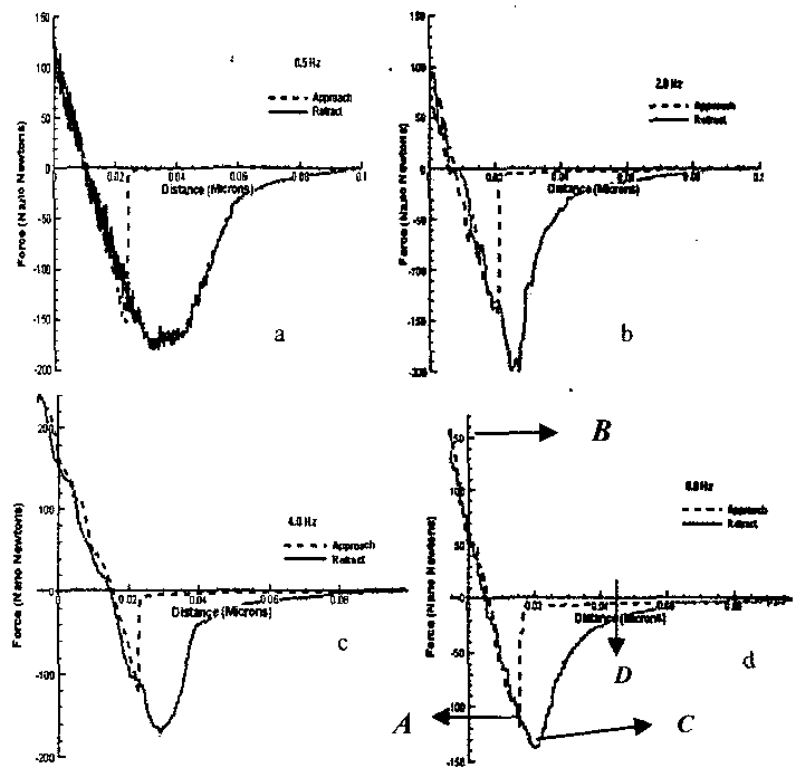

Figure 13. FD curves (a) $0.5 \mathrm{~Hz}$, (b) $2 \mathrm{~Hz}$, (c) $4 \mathrm{~Hz}$, (d) $8 \mathrm{~Hz}$

\begin{tabular}{|c|c|}
\hline Scan Rate (Hz) & Liquid bridge (Microns) \\
\hline 0.5 & 0.015 \\
\hline 2 & 0.0175 \\
\hline 4 & 0.02 \\
\hline 8 & 0.025 \\
\hline
\end{tabular}

fields, such as boundary lubrication, adhesion, polymer blends etc. The flow around small colloidal particles, thin films or capillaries [14] has been investigated. Surface Force Apparatus (SFA) has been used [14-18] in determining the nanorheology of viscoelastic fluids. Polymer solutions in a confined state exhibit increase in viscosity as the friction between the solvent and polymer molecules increases. 
Classical Force-Distance (FD) curves have been used to study the rheology at reduced lengthscales, which account for the polymer viscous-elastic forces, solid-solid adhesion, capillary forces, etc. In a typical FD experiment, the AFM probe is brought into contact with the sample. At a critical distance away from the specimen, the probe jumps and makes contact with the sample (Point A: attractive interaction), which is followed by the indentation of the specimen (Line $A B$ ). This regime is called 'Approach' and is followed by 'Retract', in which the probe is moved away from the sample. Strong adhesion forces dominate, and after the maximum adhesion force (Point C), the probe jumps back to its free state with no contact with the sample. However, in the case of polymer solutions, a thin liquid bridge is formed after Point $\mathrm{C}$, which breaks at point $\mathrm{D}$. We present results on FD curves carried on PMMA-Chlorobenzene solution (Figure 13). At low scan rates, the liquid bridge breaks early as we believe viscous forces to be dominant. At higher scan rates, the viscous forces do not have enough time to dominate and the bridge extends to greater lengths and behaves like a glassy solid. Table 1 . summarizes the bridge lengths obtained. We used a cantilever with a stiffness of $14 \mathrm{~N} / \mathrm{m}$.

\section{Fiber formation Simulations}

The mathematical modeling of flows is described by the theory of continuum mechanics. The governing equations consist of conservation laws and constitutive relationships. The conservation equations are derived from the principle of conservation of mass, the principle of balance of linear momentum, and the principle of balance of energy.

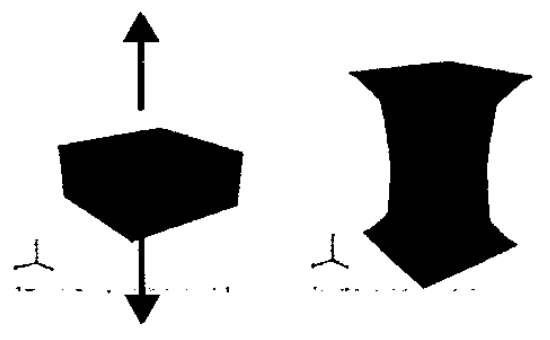

Figure 14. Extensional Defornation applied to a fluidic sample

Constitutive equations relate the stresses in the fluid to the deformation history. Flows with significant elongational components are common in industrial applications, such as fiber spinning and the food industry. Filament stretching flows are used to measure extensional viscosity in highly mobile fluids, where the extension rates may vary. Filament stretching extensional rheometers have been developed in the past [1926]. We plan on investigating the use of AFM as a micro/nano-extensional rheometer, and as a starting point in this study, we investigate the effect of different velocity profiles on pulling a viscoelastic fluid sample placed between two flat surfaces. In the filament stretching apparatus, a cylindrical liquid column is first generated between two concentric plates and then elongated by pulling one or both of the end-plate fixtures (Figure 14). It is desired that the resulting flow kinematics in the liquid column approximate an ideal uniaxial elongational flow. The extensional viscosity is determined from the axial force at the end-plate, which is measured as a function of time. The major problems with the conventional filament stretching configuration are: (i) the presence of a deformable free surface and (ii) the two rigid, non deforming end-plates. The former leads to a significant 'necking' in the central region of the liquid bridge due to surface tension of the fluid, and latter results in an appreciable shear component in the deformation history due to the no-slip boundary condition at the rigid-end plates. As a result the elongation is not shear-free, the extension rate is spatially and temporally inhomogeneous, and analysis of the experimental results does not lead to true extensional material properties, but an apparent extensional viscosity. In this preliminary report, we investigate the effect of three velocity profiles (Ramp, Exponential and Step) on the fluid deformation and the axial stress values generated within the sample. The freesurface simulations have been carried out in POLYFLOW ${ }^{\circledR}$ using the Oldroyd-B model. This is one of the simplest models for viscoelastic fluid elements. The details of this model are omitted here for the sake of brevity, but can be easily found in literature [27-30]. The choice of velocity profiles (Figure 15) ensures extensional behavior of the sample. In this study, a unit strain is applied for one second.

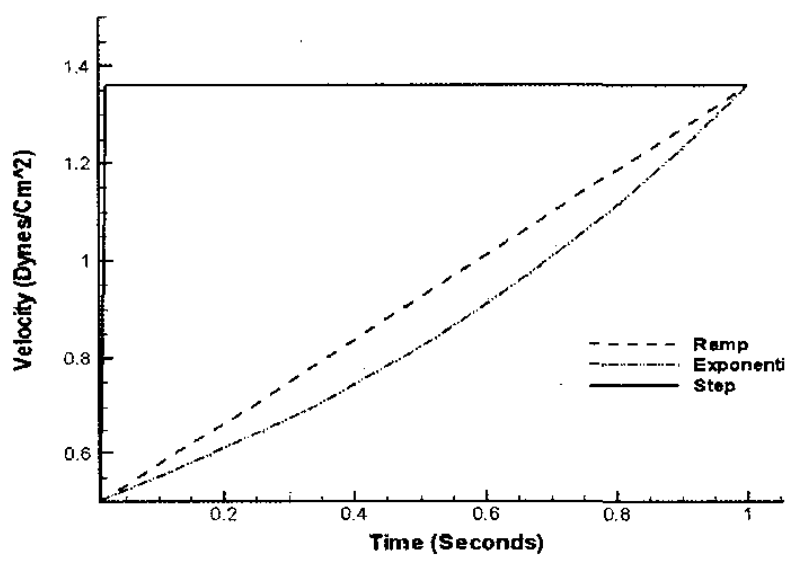

Figure 15 . Velocity profiles used in the numerical study

We have chosen the following material parameters:

$\eta$ (total viscosity) $=1000$ poise

$\lambda$ (relaxation time) $=0.1$ and 0.8 seconds

$\eta_{2} / \eta$ (Newtonian viscosity) $=0.1$

These parameters were chosen to aid in this numerical study of velocity profiles. The relaxation time $\lambda$ is defined as the time required for the shear stress to be reduced to half its original equilibrium value when the strain rate vanishes. A high $\lambda$ indicates that the memory retention of the flow is high and a low $\lambda$ signifies a fluid approaching Newtonian flow. We include the body forces and inertia effects in our numerical study. Of key interest is the thinnest cross-section of the polymer sample in the center during elongation. This would be 


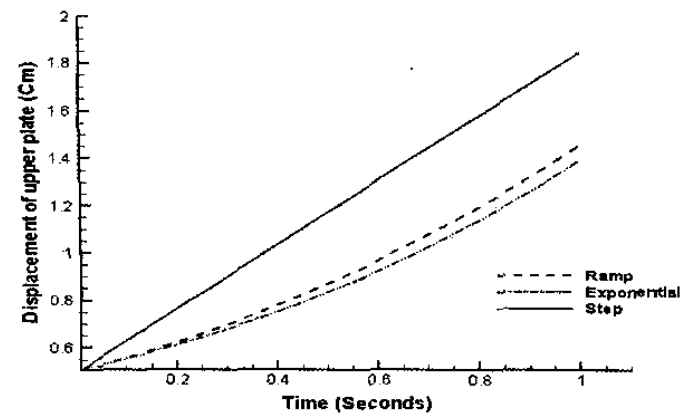

Figure 16. Displacement of upper boundary during extensionl

achieved with the velocity profile exhibiting the maximum extension (Figure 16) due to conservation of volume. The step velocity function exhibits the maximum extension and also the maximum axial stress in the fluid element (Figure 17). A comparison (Figure 18) is made between two fluid samples

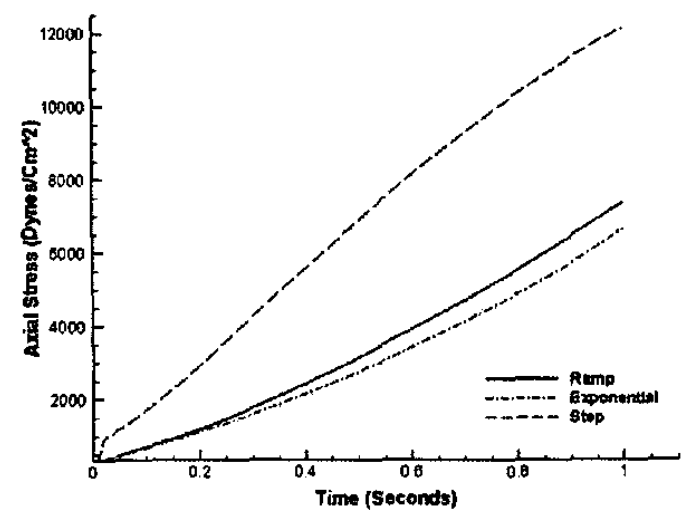

Figure 17. Axial stress at the upper boundary during extension

having relaxation times of 0.1 (approaching Newtonian behavior) and 0.8 (viscoelastic sample). The Newtonian fluid exhibits a sharp increase in the stress which is attributed to the viscous effects. Filament in Figure 18 is a point taken in

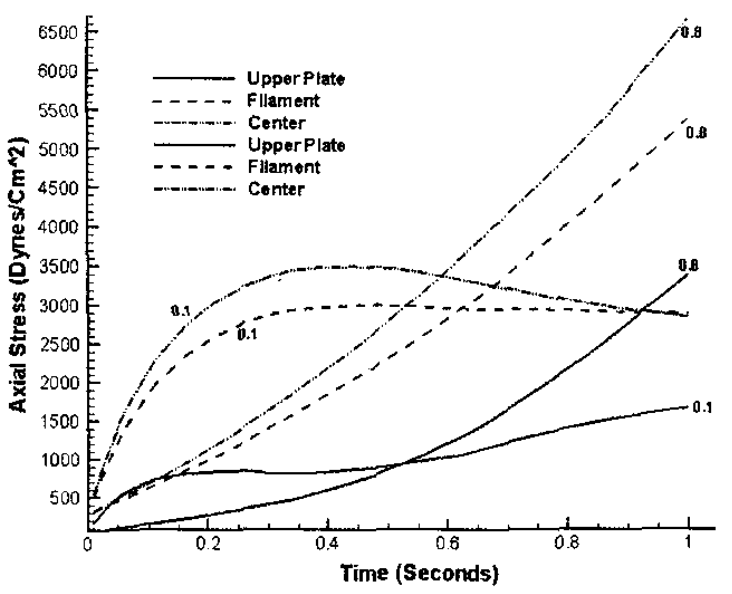

Figure 18. Comparison between Newtonian and Viscoelastic polymers between the upper plate and center. These preliminary simulations serve as a starting point in developing the appropriate control system strategy for pulling liquid polymer fibers using AFM.

\section{CONCLUSIONS}

We have successfully demonstrated the ability to fabricate extremely high aspect ratio polymer micro/nano fibers using Atomic Force Microscope. Fibers have been created in vertical, horizontal, and three-dimensional directions. A heat transfer model has been created to simulate the curing reaction of thermoset resins. Our future goals include determining the material behavior at reduced lengthscales, coupled with models developed to simulate pulling. We also envision interfacing our experiments with haptic devices. This humanmachine interface will improve upon our existing knowledge of micro/nano scale physics. We will also be working on customizing the pulling in a pre-determined three-dimensional trajectory, thus heralding new frontiers towards a reality of nano-manufacturing.

Future studies also include performing a comprehensive investigation of various polymer/solvent combinations to determine the best solution for pulling for high aspect ratio fibers. We also plan on pulling multiple fibers using customized AFM probes. Though the fibers obtained in this study are on micron scale, we are confident that nanometer scale fibers can be pulled by using STM probes along with the proper mix of polymer-solvent solution

We are also beginning to design experiments to determine the extensional viscosity of polymeric solutions at reduced lengthscales. Nano Extensional Rheometers would prove to be useful both in academia and industry. We plan on simulating the fiber pulling process using POLYFLOW ${ }^{\text {B }}$ software. This would be coupled with our experiments. Additionally, the force feedback features of AFM allow for quantitative characterization of viscoelastic properties through ForceDistance curves. We are currently in the process of generating such data for a variety of solution concentrations.

\section{ACKNOWLEDGMENTS}

The authors are thankful for the valuable suggestions made by our colleagues in our NanoRobotics Laboratory, in particular by Daniel Goldman. We would also like to acknowledge the insightful suggestions and help provided by Tomasz Kowalewski and Annette Jacobson at Carnegie Mellon University.

\section{REFERENCES}

1. Charies L. Tucker, Computer Modeling for Polymer Processing, Hanser Publishers, 1989.

2. Jean-Pierre Pascault, Thermosetting Polymers, Marcell Dekker, Inc., 2002

3. Vincenzo Capasso, Mathematical Modeling for Polymer Processing, Springer, 2002.

4. M. Sitti, "Survey of Nanomanipulation Systems," Proc. of the IEEE Nanotechnology Conference, pp. 75-80, Maui, Hawaii, 2001. 
5. M. Sitti and H. Hashimoto, "Controlled Pushing of Nanoparticles: Modeling and Experiments," IEEE/ASME Trans. on Mechatronics, vol. 5, no. 2, pp. 199-211, June 2000.

6. E. Zussman, A. Theron, and A.L. Yarin, "Formation of Nanofiber Ccrossbars in Electrospinning," Applied Physics Letters, vol. 82, no. 6 , pp. 973-5, 10 Feb. 2003.

7. J.C. Hulteen, H.X. Chen, C.K. Chambliss, and C.R. Martin, "Template Synthesis of Carbon Nanotubule and Nanofiber Arrays," Nanostructured Materials, vol. 9, pp. 133-6, 1997.

8. U. Drechsler, U. Durig, B. Gotsmann, W. Haberle, M.A. Lantz, H.E Rothuizen, R. Stutz, and G.K. Binnig, "The Millipede-Nanotechnology Entering Data Storage," IEEE Trans. on Nanotechnology, vol. 1, no. 1, pp. 39-55, March 2002

9. R.D. Piner, J. Zhu, F. Xu, S. Hong, and C.A. Mirkin, "Dip-Pen Lithography", Science, Vol. 283, p. 661-663, 1999.

10. A. Nain and M. Sitti, "3D Nanoscale Manufacturing by Nanoprobes based Controlled Pulling of Liquid Polymers," Proc. of the IEEE Nanotechnology Conference, San Francisco, August 2003.

11. M. Sitti and $\mathbf{H}$. Hashimoto, "Teleoperated Touch Feedback of Surfaces at the Nanoscale: Modeling and Experiments," IEEE/ASME Trans. on Mechatronics, vol. 8, no. 2, pp. 287-298, June 2003.

12. M. Sitti and H. Hashimoto, "Tele-nanorobotics using Atomic Force Microscope as a Robot and Sensor," Advanced Robotics, vol. 13, no. 4 pp. 417-436, 1999.

13. S.A. Harfenist; S.D. Cambron, R.S. Keynton, and R.W. Cohn, "Custom Fabrication of Freestanding and Suspended Three-Dimensional Polymer Structures", Proc. of the IEEE Nanotechnology Conference, San Francisco, August 2003.

14. F. Brochard Wyart and P. G. de Gennes, "Viscosity at Small Scales in Polymer Melts”, European Physical Journal E, 1, pp 93-97, 2000

15. Ken Nakajima, Hideki Yamaguchi,Jeong-ChangLee, Masami Kageshima, Takayuki lkehara and Toshio Nishi, "Nanorheology of Polymer Blends Invesitgated by Atomic Force Microscopy", Jpn. Journal of Applied Physics, Part 1 36, pp. 3850,1997

16. Ken Nakajim, Keita Mitsui, Atshushi lkai and Massahiko Hara, " Nanorheology of Single Protein Molecules", Riken Review, No. 37, pp. 58-62, July 2001

17. Yasuhiro Sakai, Takayuki Ikehara, Toshio Nishi, Ken Nakajima and Masahiko Hara, "Nanorheology Measurement on a Singlc Polymer Chain", Applied Physics Letters, Volume81, \#4, pp. 724-726,2002

18. M. B. Viani, T. E. Schaffer, G. T. Paloczi, L. I. Pietrasanta, B. L. Smith, J. B. Thompson, M. Richter, M Reif, H. E. Gaub, K. W. Plaxo, A. N Cleland, H. G Hansma and P. K. Hansma, "Fast Imaging and Fası Force Spectroscopy of Single Biopolymers with a New Atomic Force Micrsocope Designed for Small Cantilevers", Review of Scientific Instruments, Volume 70, Number 11, pp 4300-4303, 1999

19. J. E. Matta and R. P. Tytus, "Liquid Stretching Using a Falling Cylinder", J. of Non-Newtonian Fluid Mech., 35 (1990), pp $215-229$

20. R. Kroger, S. Berg. A. Degado, H.J. Rath, "Stretching Behavior of Large Polymeric and Newtonian Liquid Bridges in Plateau Simulation", J. Non-Newtonian Fluid Mech. 45 (1992), 385-399

21. V. Tirtaatmadja, and T. Sridhar, "A Filament Stretching Device for Measurement of Extensional Viscosity", J. of Rheology, 37(1993) $1081-1102$

22. S. Berg, R. Kroeger and H.J. Rath, "Measurement of Extensiona Viscosity by Stretching Large Liquid Bridges in Microgravity", J. NonNewtonian Fluid Mech. 55 (1994), 307-319

23. S. H. Spiegelberg and G. H. McKinley, "Stress Relaxation and Elastic Decohesion of Viscoelastic Polymer Solutions in Extensional Flow", J. Non-Newtonian Fluid Mech. 67 (1996), 49-76

24. Minwu Yao, Gareth H. McKinley and Benoit Debbaut, "Extensional Deformation, Stress Relaxation and Necking Failure of Viscoelastic Filaments", J. Non-Newtonian Fluid Mech. 79 (1998), 469-501

25. Shelly L. Anna, Chris Rogers and Gareth H. McKinley, "On Controlling the Kinematics of a Filament Stretching Rheometer using a Real-time Active Control Mechanism", J. Non-Newtonian Fluid Mech. 87 (1999) $307-335$
26. Shelly L. Anna and Gareth H. McKinley, "Elasto-Capillary Thinning and Breakup of Model Elastic Liquids", J. Rheology, 45(1), 2001, 115138

27. S. A. Khan and R. G. Larson, "Comparison of Simple Constitutive Equations for Polymer Meits in Shear and Uniaxial extensions", J. Rheology, 31(3), 1987, 207-234

28. V. Tirtaatmadja and T. Sridhar, "Comparison of Constitutive Equations for Polymer Solutions in Uniaxial Extension", J. Rheology, 3996), 1995, $1133-1160$

29. R. Byron Bird and John M. Wiest, "Constitutive Equations for Polymeric Liquids", Annual Review Fluid Mechanics, 27, 1995, 169-93

30. Polyflow User's Manual, ver. 3.10.0, Fluent Inc. 